Revue de recherche interdisciplinaire sur le genre et la sexualité

$37 \mid 2020$

Le rideau déchiré

\title{
"Le droit de cité » : sexologie, homosexualité et discours des droits de la personne dans la Pologne socialiste des années 1970
}

\section{Agnieszka Kościańska}

\section{(2) OpenEdition}

1 Journals

Édition électronique

URL : https://journals.openedition.org/sextant/336

DOI : $10.4000 /$ sextant.336

ISSN : 2795-8736

Éditeur

Éditions de l'Université de Bruxelles

\section{Édition imprimée}

Date de publication : 1 décembre 2020

Pagination : 135-148

ISBN : $978-2-8004-1744-8$

ISSN : $1370-267 X$

\section{Référence électronique}

Agnieszka Kościańska, « « Le droit de cité » : sexologie, homosexualité et discours des droits de la personne dans la Pologne socialiste des années 1970 », Sextant [En ligne], 37 | 2020, mis en ligne le 01 novembre 2021, consulté le 24 janvier 2022. URL : http://journals.openedition.org/sextant/336 ; DOI : https://doi.org/10.4000/sextant.336

\section{(c) (†) (ㅇ)}

La revue Sextant est mise à disposition selon les termes de la Licence Creative Commons Attribution Pas d'Utilisation Commerciale - Partage dans les Mêmes Conditions 4.0 International. 


\title{
« Le droit de cité » : sexologie, homosexualité et discours des droits de la personne dans la Pologne socialiste des années 1970
}

\author{
Agnieszka KoścıAŃsKA
}

En Pologne socialiste, l'homosexualité était légale ${ }^{1}$. Elle n'était pas, cependant, socialement acceptée et elle demeurait souvent invisible. On situe généralement aux années 1980 l'émergence de l'identité et des droits LGBT en Pologne, avec la publication des premiers magazines pour les homosexuel $\cdot l^{2}{ }^{2}$ et la fondation

${ }^{1}$ L'homosexualité a été dépénalisée dès 1932 dans le premier Code pénal polonais moderne. Seule la prostitution homosexuelle (contrairement à la prostitution hétérosexuelle) était pénalisée, mais cette différence de traitement disparut en 1969 quand la prostitution homosexuelle cessa d'être illégale. En outre, le Code pénal de 1932 adopta une définition large de la violence sexuelle qui faisait que le viol commis sur un individu du même sexe pouvait être poursuivi. De plus, le droit polonais n'a jamais comporté des discriminations telles que des âges de consentement différents pour les homosexuels et les hétérosexuels (M. PŁATEK, « Sytuacja osób homoseksualnych w prawie karnym », in R. WierUSZEWSKI et M. WYrZYKOwSKI (dir.), Orientacja seksualna i tożsamość płciowa, Varsovie, Instytut Wydawniczy EuroPrawo, 2009, p. 49-81). Par conséquent, la situation légale des homosexuels était bien meilleure en Pologne que dans les autres pays du socialisme d'État (voir, par exemple, F. STella, Lesbian Loves in Soviet and Post-Soviet Russia, Londres, Palgrave Macmillan, 2015 ; D. Healey, Homosexual Desire in Revolutionary Russia, Chicago, University of Chicago Press, 2001 ; L. Essig, Queer in Russia, Durham, NC, Duke University Press, 1999 ; J. McLellan, Love in the Time of Communism Intimacy and Sexuality in the GDR, Cambridge, Cambridge University Press, 2011).

${ }^{2}$ Dans le présent article, nous utilisons les termes en usage dans les années 1970-1980. C'est pourquoi, quand nous abordons ces décennies, nous employons principalement le terme « homosexuel » et nous évitons celui de " gay » puisqu'il existait à peine à l'époque en Pologne. Comme la plupart des textes de la période traitent de l'homosexualité masculine, nous consacrons notre étude à cette dernière. 
des premières associations ${ }^{3}$. Dans le présent article, nous interrogeons la période qui précède ce moment : les années 1970. Nous cherchons, en effet, à identifier les mécanismes qui ont préparé les processus d'émancipation des années 1980 et suivantes. Les publications de sexologie, sur lesquelles nous concentrons notre analyse, connaissaient alors une grande popularité et constituaient, de fait, un lieu essentiel pour discuter des questions liées à la sexualité. Nous étudions, en outre, les lettres que les sexologues recevaient et leurs réponses à ces mêmes lettres, qui étaient publiées dans la presse grand public. Il s'agit de mettre en évidence les changements significatifs qui se produisirent, au cours des années 1970, dans ces échanges entre les sexologues et leurs lecteurs. Les articles du début de la décennie insistaient sur la souffrance des homosexuels et soulignaient le caractère pathologique de leur sexualité. Au même moment, cependant, dans ces mêmes textes, les sexologues proposaient (souvent de manière détournée) des informations sur le progrès des droits homosexuels à l'Ouest. Les articles de la fin des années 1970 se montreront beaucoup plus explicites sur cette question des droits. Aussi, dans le présent article, nous cherchons à comprendre les processus qui ont suscité ce changement. Quels sont les processus qui ont conduit les sexologues et leurs écrits à contribuer au premier discours sur les droits LGBT en Pologne ${ }^{4}$ ?

En cherchant à comprendre pourquoi les sexologues ont commencé à changer leur façon d'écrire sur l'homosexualité, nous identifions deux facteurs. Il en va, d'abord, de la spécificité de la sexologie polonaise de l'époque : elle était axée sur les patients, en dialogue constant avec eux et elle se montrait ouverte aux apports des sciences humaines. Pour répondre aux préoccupations de leurs patients, les sexologues avaient recours à de nombreuses sources polonaises et allaient en chercher d'autres de l'étranger (y compris des ressources non scientifiques, comme des œuvres artistiques, de la littérature et des brochures militantes). Ils sympathisaient avec leurs patients au point de modifier leur perspective scientifique. Cependant, le contexte politique et social a souvent limité l'ampleur du changement que ce premier facteur a pu apporter. Aussi, dans un second temps, nous considérons, comme autre facteur,

${ }^{3}$ B. Warkocki, « Trzy fale emancypacji homoseksualnej w Polsce », Porównania, 15, 2014, p. 121-132 ; L. SzulC, Transnational Homosexuals in Communist Poland, New York, Palgrave Macmillan, 2018 ; A. Fiedotow, « Początki ruchu gejowskiego w Polsce », in M. Kula (dir.), Kłopoty z seksem w PRL. Rodzenie nie calkiem po ludzku, aborcja, choroby, odmienności, Warszawa, Wydawnictwa Uniwersytetu Warszawskiego, Instytut Pamięci Narodowej, 2012, p. 271-272.

${ }^{4}$ Cette recherche a pu être menée grâce au projet « Cruising the 1970s: Unearthing PreHIV/AIDS Queer Sexual Cultures » (CRUSEV). Le projet CRUSEV bénéficie du soutien financier du Programme commun de recherche HERA (www.heranet.info), cofinancé par le NCN, le BMBF via le DLR-PT, le MINECO, l'AHRC et la Commission européenne via Horizon 2020. Tous mes remerciements vont aux membres de l'équipe polonaise du CRUSEV pour les documents qu'ils ont portés à ma connaissance et pour leurs suggestions. Une version antérieure de cet article a été présentée au colloque international « Sexologie et idéologie à l'âge de l'institutionnalisation (1960-2000) », Université libre de Bruxelles, 21-23 mars 2018. Je voudrais remercier les organisateurs de la conférence pour leur invitation et tous les participants pour leur contribution. 
les changements qui se sont précisément produits dans ce même contexte. Selon nous, la formulation explicite du concept de droits homosexuels n'était pas possible sans les nouvelles dynamiques sociales qu'ont généré la reconfiguration des interactions Est/Ouest, à la suite des accords d'Helsinki de 1975. Les accords d'Helsinki et l'établissement de la CSCE (Conférence sur l'organisation de la sécurité et de la coopération en Europe) provoquèrent, en effet, une prolifération de discours sur les droits de la personne. Dans cette perspective, nous nous appuyons sur les travaux de Gunter Dehnert, un historien allemand qui a montré que l' " effet Helsinki » a été particulièrement important en Pologne. Nous montrons que, même si les droits liés à la sexualité n'étaient pas mentionnés dans l'accord final d'Helsinki, ce dernier n'en a pas moins eu de grandes conséquences sur les droits des homosexuel·les. Notamment, le discours sur les droits de la personne a progressé et le Parti communiste a hésité à intervenir pour l'empêcher de croître plus encore. L' « effet Helsinki » a ainsi ouvert un espace où il est devenu possible de parler ouvertement des droits sexuels ${ }^{5}$.

Le présent article se fonde sur notre recherche dans les archives sur la sexologie polonaise (des années 1970 à nos jours), effectuée à partir d'un corpus constitué des principales publications de sexologie universitaires, des manuels populaires d'information ou d'éducation sexuelle, des magazines qui proposaient une rubrique consacrée à la sexualité et des lettres adressées aux sexologues. Nous avons également mené des interviews approfondies auprès de sexologues qui étaient en activité dans la période qui nous intéresse. Enfin, nous avons discuté des publications sexologiques de l'époque avec des membres de la communauté LGBTQ qui avaient vécu les années 1970 - il s'agit principalement de membres d'un groupe de soutien LGBTQ pour les seniors qui dépend d'une organisation LGBTQ polonaise.

\section{Des lettres et des réponses}

« Je suis quelqu'un de malheureux. Je n'arrive pas à étudier, j'ai perdu l'envie de vivre, je ne crois ni en moi ni en les autres. Tout va très mal pour moi. La cause de tout cela, c'est l'homosexualité... Je me dégoûte. Je veux devenir quelqu'un de normal. $»^{6}$ Voilà ce que G., un habitant des alentours de Wroclaw, écrivait dans une lettre à un sexologue. Le sexologue en question, Zbigniew Lew-Starowicz, qui tenait une rubrique consacrée à la sexualité dans un hebdomadaire étudiant progressif et très populaire appelé Itd (Etc.), de 1969 à 1990, cita cette lettre, avec d'autres lettres similaires d'homosexuels, dans son article de 1970 intitulé Homoseksualizm («Homosexualité »). Il ouvrait son article avec une synthèse des lettres qu'il avait reçues : «Malgré certaines différences dans les causes de leur homosexualité et la manière dont elle s'est manifestée, les auteurs de ces lettres sont, fondamentalement, d'accord. Ils voient dans l'homosexualité quelque chose d'immoral, ils se considèrent eux-mêmes comme des malades, et ils veulent être soignés. Certaines lettres portent le témoignage de l'authentique souffrance de leurs auteurs. ${ }^{7}$ Les lettres citées dans

${ }^{5}$ G. Dehnert, « The Polish Opposition, the Crisis of the Gierek Era, and the Helsinki Process ", in J. EcKel et S. Moyn (dir.), The Breakthrough Human Rights in the 1970s, Philadelphia, University of Pennsylvania Press, 2014, p. 166-185.

${ }^{6}$ Cité par Z. Lew-StarowicZ, « Homoseksualizm », Itd, 8, 1970, p. 14.

${ }^{7}$ Ibid. 
cet article avaient été sélectionnées de façon significative : elles décrivaient toutes la souffrance des homosexuels et la discrimination qu'ils subissaient. En 1973, LewStarowicz commentait d'autres lettres que des homosexuels lui avaient envoyées : «Les auteurs de ces lettres, dans leur majorité, demandent la "réhabilitation" de l'homosexualité afin qu'elle soit considérée comme une façon normale de satisfaire ses besoins sexuels; certains demandent des informations sur les causes, les symptômes et les conséquences de l'homosexualité et des autres formes de perversion. ${ }^{8}{ }^{8}$ À toutes ces lettres, Lew-Starowicz adressait une réponse claire : «Un traitement est possible et il est efficace. ${ }^{9}{ }^{9}$

Mais, si nous regardons plus attentivement sa réponse, nous voyons qu'en fait, sa réaction est plus complexe. Dans le même article, il décrivait la vie homosexuelle à l'étranger et il indiquait l'émergence de communautés homosexuelles : «L'homosexualité est l'un des troubles les plus fréquents du développement et de l'orientation du désir sexuel. Ce phénomène apparaît dans de nombreux pays et, dans certains, il a même gagné le droit de cité grâce à des procédures pénales indulgentes, des magazines et des discothèques réservés aux homosexuels, ainsi que des cafés, des plages et des associations diverses. $"{ }^{10} \mathrm{Il}$ commentait, en outre, les résultats de recherches menées en Amérique du Nord qui prouvaient que l'homosexualité n'était pas une maladie. Ces recherches, quelques années plus tard, mèneraient à sa dépathologisation aux États-Unis ${ }^{11}$. Il écrivait, par exemple : «Certains chercheurs, étonnés par l'ampleur massive du phénomène, ont essayé de le situer dans le champ des normes psychosexuelles. Dans leurs travaux, ils mentionnent des phénomènes similaires chez certaines espèces animales. $"{ }^{12}$ Ce faisant, il prenait ses distances par rapport à ces chercheurs : «Il est difficile d'être en accord avec ces suggestions. Dans la vie sexuelle animale, c'est un problème marginal qui se produit principalement en captivité, il s'agit donc, alors, d'une simple forme de substitution. ${ }^{13}$ Pour des lecteurs qui étaient habitués à lire entre les lignes, le message était clair : c'était là une façon, pour Lew-Starowicz, d'éviter l'intervention de la censure ${ }^{14}$, tout en déstigmatisant l'homosexualité en l'associant à l'Ouest, à cet Ouest éminemment désirable, où les

${ }^{8}$ Z. Lew-StarowicZ, « Listy do seksuologa (2)», Itd, 10, 1973, p. 20.

9 Z. Lew-StarowicZ, « Homoseksualizm », op. cit.

${ }^{10}$ Ibid.

${ }^{11}$ C'est-à-dire les recherches menées par Alfred Kinsey, Evelin Hooker, Cleland Ford et Frank Beach ; voir R. BAYER, Homosexuality and American Psychiatry: The Politics of Diagnosis, Princeton, NJ, Princeton University Press, 1981.

12 Z. Lew-StarowicZ, « Homoseksualizm », op. cit.

13 Ibid.

${ }^{14}$ En Pologne socialiste, chaque article devait être vérifié par un censeur avant publication afin de contrôler sa conformité avec la doctrine socialiste, et, dans ce cas précis, sa moralité. Lew-Starowicz excellait à tromper les censeurs. Il nous a dit, ce qu'il explique également en détail dans son autobiographie, que la censure n'était intervenue dans ses articles pour Itd que trois fois en 20 ans (Z. Lew-Starowicz, Pan od seksu. Autobiografia najslynniejszego polskiego seksuologa, Cracovie, Znak, 2013 ; A. KoścıaŃsKa, Płeć, przyjemność i przemoc. Ksztaltowanie wiedzy eksperckiej o seksualności w Polsce, Warszawa, Wydawnictwa Uniwersytetu Warszawskiego, 2014, p. 82 ; sur la censure et l'homosexualité, voir L. SzulC, op. cit., p. 101). 
homosexuels possédaient « le droit de cité », des magazines, des discothèques et des associations.

La lecture de la rubrique de Lew-Starowicz montre que, quelques années plus tard, ce genre de précaution n'était plus nécessaire. Le ton de ses écrits sur l'homosexualité changea alors complètement. Son article de 1978, intitulé Inaczej (« Différemment»), commence par une longue citation de la lettre d'Igor : « D’abord, c'était innocent. Après avoir perdu ma petite amie - un verre de cognac, un homme jeune et beau, ivre d'alcool et de camaraderie; une invitation à passer la nuit ensemble... Au matin, de la confusion; c'était si différent de toutes ces nuits passées avec des femmes. Puis plusieurs autres contacts, généralement riches en plaisir. $\gg{ }^{15}$ Igor poursuit en décrivant sa situation : " De temps en temps, je succombe à la tentation de rencontrer un homme. C'est facile de la façon dont je procède. Cette échappée dans un autre monde est presque comme le parachèvement de mon bonheur. Ces rendez-vous secrets avec divers jeunes gens me donnent accès à une variété d'expériences sexuelles, sociales, cognitives et psychologiques. $\gg{ }^{16}$ Igor parle ouvertement de ses droits et de ses besoins ainsi que de ceux des autres homosexuels : «Il y a beaucoup d'hommes comme moi, ce que j'ai pu souvent constater... Chacun d'entre nous a droit [à ce que] nous trouvons beau... N'est-il pas temps que ces barrières tombent ? $\gg{ }^{17}$ Dans sa réponse à la lettre d'Igor, Lew-Starowicz soutient l'idée de droits pour les homosexuels et exhorte la société à les respecter ${ }^{18}$. Il n'était alors plus besoin de lire entre les lignes : la lettre d'Igor constitue la première description directe de la vie homosexuelle, associée à une demande de droits pour la communauté homosexuelle masculine, à être publiée dans la presse généraliste en Pologne. Cela se produisit avant l'émergence du mouvement pour les droits des homosexuel·les.

Aussi, pour reformuler la problématique principale de cet article, dans ce qui suit, nous essayons de comprendre ce qui s'est passé entre 1970 et 1978 qui permit à Lew-Starowicz de publier la lettre d'Igor et de parler ouvertement des droits des homosexuel-les.

\section{Une sexologie axée sur le patient}

Pourquoi les sexologues ont-ils commencé à tenir, dans leurs écrits, un discours différent sur l'homosexualité à la fin des années 1970? Cela pourrait s'expliquer, en partie, par la nature de la sexologie polonaise, axée sur le patient et ouverte aux apports des sciences humaines. Comme nous l'avons expliqué ailleurs ${ }^{19}$, dans leurs écrits, les sexologues polonais se montraient ouverts aux besoins et aux préoccupations de leurs patients et de leurs lecteurs. Ils les replaçaient dans un contexte socioculturel plus vaste. Ils croyaient, en effet, qu'un sexologue devait resituer les problèmes de leurs

\footnotetext{
15 Cité par Z. Lew-Starowicz, « Inaczej », Itd, 52-53, 1978, p. 30.

16 Cité in ibid.

17 Cité in ibid.

18 Ibid.
}

19 A. KościańsKa, op. cit., A. KościańskA, « Beyond Viagra: Sex Therapy in Poland », Sociologický časopis/Czech Sociological Review, 50/6, 2014, p. 919-938 ; A. KościańsKA, "Sex on equal terms? Polish sexology on women's emancipation and "good sex" from the 1970s to present », Sexualities, 19/1-2, 2016, p. 236-256. 
patients et patientes dans la perspective plus large du social, du culturel, du politique et du psychologique. Comme l'expliquait le fondateur de la sexologie polonaise de l'après-guerre, Kazimierz Imieliński : «On croit souvent que la sexologie est une discipline très spécialisée. C'est une idée complètement fausse. À l'évidence, la sexologie est étroitement liée à des disciplines médicales telles que la psychiatrie, la neurologie, l'urologie... Mais remarquez également les liens qui existent entre elle et des disciplines qui n'appartiennent pas aux sciences naturelles : la pédagogie, le droit, la psychologie, et l'on pourrait même soutenir qu'elle est liée à la théologie. ${ }^{20}$ Imieliński et d'autres sexologues de la fin de l'ère socialiste avaient construit leurs connaissances et leur pratique à partir d'études de cas de leurs patients. Des textes sexologiques très populaires regorgeaient de témoignages personnels. Dans les années 1970-1980, les sexologues se concentraient sur les réponses à apporter aux préoccupations de leurs patients. La rubrique de Lew-Starowicz dans Itd constitue l'exemple le plus manifeste de ce dialogue. Elle comprenait généralement de longues citations de lettres reçues par Lew-Starowicz et les réponses qu'il leur avait apportées. Dans ses réponses, le sexologue faisait appel à plusieurs types de solutions pour aider ses lecteurs. Par exemple, une femme écrivait à Lew-Starowicz : « D'abord, je vais au travail, puis je rentre à la maison, je m'occupe des tâches domestiques, le soir venu, je suis épuisée et je n'ai qu'une envie - aller dormir, dormir le plus longtemps possible. $\gg{ }^{21}$ Cette lettre ouvrait une discussion sur la sexualité, sur l'émancipation des femmes et sur leur double fardeau qui leur incombait du fait des politiques du genre souvent contradictoires du socialisme d'État. D'une part, l'État incitait les femmes à travailler, mais, de l'autre, il n'encourageait pas une nouvelle division genrée du travail domestique, ce qui faisait peser un double fardeau sur les épaules des femmes. Des voix de femmes comme celle-là faisaient conclure à Lew-Starowicz que le temps libre était nécessaire pour permettre aux femmes d'accéder au plaisir sexuel, que les hommes devaient prendre part au travail domestique, mais, en même temps, il conseillait aux femmes de travailler à mi-temps. Lew-Starowicz suivait les préoccupations de ses patientes, tout en situant sa réponse dans le contexte des politiques sociales du genre : même si la majorité des femmes travaillaient à l'extérieur, l'État s'inquiétait de la baisse du taux de natalité et cherchait à faire sortir les femmes du marché du travail.

Les mêmes mécanismes étaient à l'œuvre en ce qui concerne l'homosexualité. Les sexologues élaboraient leurs théories en réaction aux expériences de leurs patients/lecteurs et ils s'inspiraient des nombreuses sources à leur disposition. Ils s'appuyaient ainsi à la fois sur le discours sexologique polonais et sur leurs échanges, à l'international, avec des universitaires et des militants. Sous le communisme, la mobilité des citoyens était limitée : voyager librement, et en particulier à l'étranger, n'était pas possible. Le courrier international était contrôlé par les autorités. Mais il y avait quelques exceptions. Comme Lew-Starowicz nous l'a dit pendant une interview, les sexologues, et les scientifiques en général, avaient de nombreuses occasions

${ }^{20}$ S. KRYSKA, « Kilka słów o seksuologii [an interview with Kazimierz Imieliński] », Itd, 24, 1967, p. 10, cité in A. KościańsKA, « Beyond Viagra », op. cit., p. 926.

${ }^{21}$ Z. Lew-Starowicz, «Eros i emancypacja », Itd, 49, 1970, p. 14, cité in A. KościaŃsKA, «Sex on equal terms?», op. cit., p. 248. 
de voyager à l'étranger, d'acheter des livres et de recevoir d'autres documents, par exemple des brochures militantes pour les droits homosexuels. C'est pourquoi ils étaient au courant des mouvements pour les droits des homosexuel-les. Par conséquent, dans les années 1970, ils pouvaient écrire sur les droits des homosexuel·les à l'Ouest et discuter dans le détail des travaux de sexologie, des rapports Kinsey, par exemple, qui contribuèrent à la dépathologisation de l'homosexualité à l'Ouest. La transmission d'informations de cet ordre relevait d'une stratégie délibérée de la part des sexologues. Au début des années 1980, un sexologue polonais envoya une lettre à Andrzej Selerowicz, un militant homosexuel polonais vivant en Autriche qui participait activement à l'Homosexuelle Initiative Wien (HOSI) ${ }^{22}$. Dans cette lettre, il écrivait qu'en présentant la vie homosexuelle telle qu'elle s'organisait à l'étranger, il contribuait, d'un point de vue sexologique, à l'amélioration de la vie homosexuelle en Pologne ${ }^{23}$. On ne peut pas, cependant, complètement expliquer le changement survenu dans les écrits sexologiques sur l'homosexualité par la seule ouverture d'esprit des sexologues aux problèmes de leurs patients et par l'attention qu'ils prêtaient à l'évolution de leurs préoccupations et de celle de leurs correspondants/lecteurs. Au début des années 1970, dans leurs écrits, les sexologues se heurtaient à d'importantes contraintes. L'homosexualité était perçue comme une atteinte à la moralité socialiste et toutes les organisations issues de la société civile (comme les associations pour les droits des homosexuel $\cdot \mathrm{les}^{24}$ ) étaient interdites sous le socialisme. Les sexologues devaient tenir compte du contexte politique et, s'ils voulaient voir leurs textes publiés, ils ne pouvaient pas transgresser les lois de la moralité hétéronormative socialiste. Par conséquent, d'autres facteurs doivent être pris en compte pour expliquer ce changement.

\section{Droits des homosexuels et « effet Helsinki »}

On peut également attribuer ces changements dans les publications de sexologie destinées au grand public à une modification générale de la façon de penser les droits de la personne. Cette relation de causalité n'est cependant pas aussi évidente que dans d'autres contextes politiques, puisque l'opposition démocratique polonaise ne soutenait pas les droits des homosexuels ${ }^{25}$, contrairement à ce que faisait, par exemple,

${ }^{22}$ Sur l'HOSI, voir L. Szulc, op. cit. ; A. Fiedotow, op. cit. ; A. Kurimay et J. TakÁcs, " Emergence of the Hungarian homosexual movement in late refrigerator socialism ", Sexualities, 20/5-6, 2017, p. 585-603.

${ }^{23}$ Homosexuelle Initiative Wien's archive. Cette lettre nous a été signalée par Tomasz Basiuk (équipe CRUSEV).

${ }^{24}$ Ce n'est qu'à la fin des années 1980 qu'elles furent autorisées quand on toléra que les homosexuels s'organisent, tant qu'il s'agissait de prévention du VIH-sida. Voir, par exemple, L. Szulc, op. cit. ; J. OwczarzaK, « Defining HIV Risk and Determining Responsibility in Postsocialist Poland », Medical Anthropology Quarterly, 23/4, 2009, p. 417-435. Sur la dynamique similaire de la Hongrie, voir A. KuRIMAY et J. TAKÁCS, op. cit.

${ }_{25}$ Selon une femme trans, qui, dans le passé, avait été un acteur (masculin) important de l'opposition au communisme, l'opposition démocratique ne s'intéressait pas aux droits LGBT. Elle-même n'avait jamais révélé sa transsexualité à quiconque du milieu de l'opposition démocratique. À l'époque, elle estimait que les homosexuels ne pouvaient pas appartenir à l'opposition à cause de leur vulnérabilité puisque la police secrète aurait pu facilement exercer 
le mouvement contre l'apartheid en Afrique du Sud ${ }^{26}$. De même, l'antenne polonaise de Radio Free Europe ne parlait pas de sujets liés à l'homosexualité comme pouvait le faire son antenne hongroise ${ }^{27}$. Les chercheurs situent, généralement, l'émergence du discours sur les droits homosexuels en Pologne dans les années 1980. Nous estimons, quant à nous, qu'un changement général de mentalité en ce qui concerne les droits de la personne se produisit en Pologne dans la deuxième moitié des années 1970 et que ce changement mena à l'émergence du concept de droits des homosexuels. Ce changement résulte des accords d'Helsinki de $1975^{28}$ et fait partie de ce que les historiens appellent l'« effet Helsinki » ${ }^{29}$. Dans son analyse de l'opposition démocratique en Pologne dans les années 1970, Dehnert soutient l'idée que ce « premier véritable mouvement d'opposition au régime dans un pays socialiste $»^{30}$ se produisit non seulement du fait de « la logique interne de l'histoire nationale polonaise », comme le pensent en général les Polonais, mais aussi grâce aux « droits universels de la personne qui ont servi de liant entre les différentes tendances de l'opposition ${ }^{31}$. Aussi Dehnert reliet-il le développement de l'opposition anticommuniste à la prolifération généralisée du discours des droits de la personne, prolifération qui se produisit dans un contexte politique très spécifique.

En 1970, Edward Gierek devint le premier secrétaire du Parti communiste polonais. À la différence des dirigeants communistes polonais précédents, il n'était pas étroitement lié à l'URSS. Au contraire, il avait passé sa jeunesse en France et en Belgique et il était, de manière générale, ouvert d'esprit à l'égard de l'Ouest. Du fait de son parcours et du climat politique particulier de détente de l'époque, sous sa

un chantage sur eux. Aussi, elle avait elle-même activement contribué à exclure les homosexuels des cercles de l'opposition démocratique (interview menée par Karolina Morawska, projet CRUSEV).

${ }^{26}$ D. L. Donham, « Freeing South Africa: The "Modernization" of Male-Male Sexuality in Soweto », Cultural Anthropology, 13/1, 1998, p. 3-21.

27 A. KurimaY et J. TaKÁCS, op. cit.

${ }^{28}$ Les accords d'Helsinki désignent des accords internationaux qui visaient à l'amélioration des relations entre l'Ouest et l'Est communiste. Ils ont été signés à la fin de la Conférence sur la sécurité et la coopération en Europe (CSCE). Cette conférence changea de nom en 1995 et adopta celui d'Organisation pour la sécurité et la coopération en Europe (OSCE). Les accords, signés par 35 pays, déclarent que « [1]es États participants respectent les droits de l'homme et les libertés fondamentales, y compris la liberté de pensée, de conscience, de religion ou de conviction pour tous, sans distinction de race, de sexe, de langue ou de religion ». Conférence sur la sécurité et la coopération en Europe, Acte final, 1975. En ligne : https://www.osce.org/ helsinki-final-act (consulté le 4 août 2019). (Voir également G. DeHnerT, op. cit.).

${ }^{29}$ Voir, par exemple, S. Moyn, « The Return of the Prodigal: The 1970s as a Turning Point in Human Rights History », in J. Eckel et S. Moyn (dir.), The Breakthrough Human Rights in the 1970s, Philadelphia, University of Pennsylvania Press, 2014, p. 10. Moyn y écrit qu'après les accords d'Helsinki, « [p]ersonne - pas même le plus enthousiaste des Européens de l'Ouest - n'avait prévu l'explosion du nombre d'appels de dissidents aux droits de la personne qu'ont provoquée les accords ». Il est à noter que ce phénomène a été particulièrement important en Pologne.

${ }^{30}$ G. DEHNERT, op. cit., p. 168.

${ }^{31}$ Ibid. 
direction, la Pologne s'ouvrit davantage à l'Ouest, gagnant « [un] incroyable degré d'ouverture dans la sphère culturelle $\gg^{32}$ et s'orientant davantage vers le consumérisme. Les biens de consommation occidentaux, qui n'avaient pas été disponibles pendant les deux premières décennies du socialisme d'État, firent leur apparition dans les magasins polonais et, avec eux, survint un changement idéologique général. Ainsi que Dehnert le note, « cette conception d'un socialisme, axé sur la consommation, que Gierek présentait comme remède à tous les problèmes sociétaux depuis décembre $1970[\ldots]$ ne fit qu'affaiblir davantage le lien déjà ténu de la Pologne avec l'idéologie socialiste $\gg{ }^{33}$.

Gierek n'aurait pu développer cette conception sans la coopération avec l'Ouest ni sans les prêts de l'Ouest qui renforcèrent l'effet d'Helsinki. En effet, « les accords d'Helsinki, pour la première fois, établirent un lien officiel entre le degré de coopération économique avec l'Ouest et le respect des droits de la personne en Pologne. Chaque fois que la situation en matière d'approvisionnement se dégrada, comme ce fut le cas à partir de 1975, la pression sur le gouvernement polonais s'accrut de façon proportionnelle. Répondre à cette pression avec une répression exagérément violente aurait nui à la réputation du pays en ce qui concerne les droits de la personne et aurait pu pousser les pays occidentaux à retirer leurs prêts et leur aide économique $»^{34}$.

La Pologne et les autres pays de socialisme d'État d'Europe centrale étaient, dans l'ensemble, désireux de ratifier les accords d'Helsinki. L'Allemagne de l'Est, par exemple, présentait les droits de la personne comme étant étroitement liés au socialisme ${ }^{35}$. La Pologne, quant à elle, affirmait que les accords trouvaient leur origine dans ses propres efforts antérieurs pour la paix mondiale et le désarmement, c'est-àdire dans le Plan Ratacki ${ }^{36}$.

${ }^{32}$ Ibid.

33 Ibid.

${ }^{34}$ Ibid., p. 170.

35 Selon le discours dominant de la guerre froide, les droits de la personne seraient liés au capitalisme et à l'Ouest démocratique. Dans cette perspective, les droits de la personne seraient arrivés dans l'Est communiste par l'Ouest (les États-Unis et l'Europe de l'Ouest). Les recherches actuelles, cependant, font apparaître le lien entre les droits de la personne et le socialisme. Ned Richardson-Little, par exemple, a récemment montré que de nombreux intellectuels de l'Allemagne de l'Est, comme le juriste Karl Polak, pensaient que « sous le socialisme, les véritables droits de la personne pouvaient enfin prospérer à la place du simulacre de droits colportés par l'Allemagne de l'Ouest» (N. RichaRDSON-LitTLE, « Dictatorship and Dissent: Human Rights in East Germany in the 1970s », in J. Eckel et S. MoYN (dir.), The Breakthrough Human Rights in the 1970s, Philadelphia, University of Pennsylvania Press, 2014, p. 51). De même, des chercheures comme Cielia Donert et Kristen Ghodsee ont montré le profond engagement des pays socialistes dans la lutte pour les droits des femmes. K. GHODSEE, «Rethinking State Socialist Mass Women's Organizations. The Committee of the Bulgarian Women's Movement and the United Nations Decade for Women, 1975-1985 », Journal of Women's History, 24/4, 2012, p. 49-73 ; C. DonERT, « Whose Utopia? Gender, Ideology, and Human Rights at the 1975 World Congress of Women in East Berlin », in J. Eckel et S. MoYN (dir.), The Breakthrough Human Rights in the 1970s, Philadelphia, University of Pennsylvania Press, 2014, p. 68-87.

${ }^{36}$ G. DEHNERT, op. cit., p. 175. 
Selon Dehnert, « [c]'est la politique de Gierek, tant au niveau de la situation économique qu'à celui de la diplomatie internationale, qui a créé les conjectures dans lesquelles l'effet Helsinki devint envisageable. Son action a clairement signalé que la Pologne regardait au-delà du bloc de l'Est dans la seconde moitié de la décennie, sous l'effet conjugué de la dépendance économique et financière et des débuts du processus de la CSCE. C'est là la principale raison pour laquelle le gouvernement polonais a hésité à réprimer l'opposition, qui pourtant ne cessait de croître à vue d'œil ${ }^{37}$.

L'accord final d'Helsinki demandait que les «États respectent les droits de l'homme et les libertés fondamentales, y compris la liberté de pensée, de conscience, de religion ou de conviction pour tous $»^{38}$. Pour Dehnert, cet accord conduisit au développement d'une opposition anticommuniste qui s'appuyait sur les droits de la personne. Notre propre recherche sur la façon dont l'homosexualité était perçue dans les années 1970 montre que cet accord eut des conséquences plus importantes encore. L'accord final d'Helsinki a, en effet, généré une expansion du discours des droits de la personne, ce qui a donné aux sexologues plus de liberté pour aborder ouvertement la question des droits liés à la sexualité. C'est pourquoi, en 1978, Lew-Starowicz a pu publier la lettre où Igor demandait des droits pour « les hommes comme lui », alors qu'auparavant, le sexologue ne pouvait que citer des lettres qui témoignaient de la souffrance homosexuelle.

Bien que dans les années 1970, le CSCE n'abordait pas les droits des homosexuel·les en tant que droits de la personne, c'était le cas en Europe de l'Ouest et en Amérique du Nord à la fin des années 1960, au début du mouvement pour les droits des homosexuel·les. Bien que les sexologues polonais aient déjà eu connaissance du concept de droits des homosexuel·les au début des années 1970 (et bien qu'ils aient été en contact avec des organisations homosexuelles), ils ne pouvaient pas les évoquer directement dans leurs écrits. Plus encore, ils devaient même marquer une nette distance. À la fin des années 1970, grâce à la reconnaissance du concept de droits de la personne, ils purent évoquer plus ouvertement l'homosexualité. Dans la décennie suivante, certains sexologues firent pression pour la création d'une association homosexuelle ${ }^{39}$ et les homosexuels eux-mêmes invoquaient plus fréquemment le concept de droits de la personne quand ils plaidaient pour une meilleure acceptation par la société ${ }^{40}$. L'effet Helsinki ne peut être limité au progrès de l'opposition démocratique. Il nous faut aussi prendre en compte ce phénomène général de prolifération du discours des droits de la personne et ses conséquences sur des domaines qui n'étaient pourtant pas mentionnés dans l'acte final d'Helsinki.

Un autre facteur fut également agissant : l'alliance entre l'opposition anticommuniste et l'Église catholique. Auparavant, les opposants avaient pour

\footnotetext{
${ }^{37}$ Ibid., p. 169.

${ }_{38}$ Ibid., p. 174 ; voir également Conférence sur la sécurité et la coopération en Europe, op. cit.

${ }^{39}$ L. SzulC, op. cit.

${ }^{40}$ Cela apparaît nettement, par exemple, dans les lettres envoyées à l'Homosexuelle Initiative Wien au début des années 1980, comme Tomasz Basiuk l'a montré dans sa contribution à la journée d'étude « Różowy język/Pink Tongue », qui a eu lieu à l'Université de Varsovie le 9 juin 2018.
} 
objectif de reformer le socialisme, mais les événements de mars $1968^{41}$ en Pologne leur firent prendre conscience de la « vraie nature du communisme $»^{42}$ et provoquèrent le « changement de mentalité ${ }^{43}$ qui aboutit à la naissance d'un « mouvement qui parviendra[it] enfin dans les années 1970 à toucher les forces traditionnelles de la société polonaise et, tout particulièrement, les tenants du catholicisme politique $»^{44}$. Ce mouvement, c'est-à-dire l'opposition démocratique, partageait avec l'Église catholique un « engagement à défendre les droits humains élémentaires et les droits civiques $\rangle^{45}$. Il n'y avait, cependant, dans le cadre de cette alliance, nulle place pour les droits sexuels. C'était manifeste, par exemple, dans un débat sur la sexualité qui eut lieu, dix ans plus tard, dans Tygodnik Mazowsze, le principal magazine clandestin (samizdat) de l'opposition. Un article, qui adoptait un point de vue progressiste sur la sexualité et qui s'opposait à l'Église catholique au sujet d'un manuel d'éducation sexuelle, provoqua la colère des lecteurs ${ }^{46}$. Ce manuel comprenait, par exemple, un chapitre sur l'homosexualité qui présentait les relations homo- et hétérosexuelles comme étant $«$ analogues $»{ }^{47}$. Les lecteurs estimaient en effet que la sexualité n'avait rien à faire dans Tygodnik Mazowsze puisqu'il s'agissait d'un magazine politique. De plus, ils reprochaient à ses rédacteurs de chercher à détruire l'alliance entre l'Église catholique et l'opposition communiste ${ }^{48}$.

Depuis cette époque, cette alliance n'a cessé de jouer un rôle majeur dans la politique polonaise, ce qui a eu des conséquences directes sur la question des droits LGBTQ dans ce pays ${ }^{49}$. Par exemple, au milieu des années 1990, lors des débats sur la rédaction de la Constitution de la Troisième République, des militants liés à la Fondation Helsinki ${ }^{50}$ firent pression pour que l'orientation sexuelle figure dans le paragraphe consacré à la lutte contre les discriminations. Ils parvinrent à obtenir un large soutien, y compris celui du légendaire dirigeant de Solidarność, Lech Wałęsa. Après l'intervention de l'Église, Wałęsa retira son appui ${ }^{51}$. Aujourd'hui encore, les

${ }^{41}$ C'est-à-dire la propagande communiste antisémite qui avait pris pour cible les communistes d'origine juive qui voulaient réformer le socialisme.

${ }^{42}$ G. DehnerT, op. cit., p. 172.

43 Ibid.

${ }^{4}$ Ibid.

45 Ibid.

46 A. DodzIUK, «W obronie zdrowego rozsądku », Tygodnik Mazowsze, 227, 1987, p. 4. Lettres publiées dans les numéros 229 et 230 du magazine.

${ }^{47}$ W. Sokoluk, D. Andziak et M. Trawińska, Przysposobienie do życia $w$ rodzinie, Varsovie, Wydawnictwa Szkolne i Pedagogiczne, 1987.

${ }^{48}$ A. KościańsKa, Zobaczyć tosia. Historia polskiej edukacji seksualnej od pierwszej lekcji do internetu, Wołowiec, Czarne, 2017.

49 De même qu'elle a eu des conséquences sur d'autres questions, comme celle des droits en matière de procréation. Voir par exemple J. Mishtal, The Politics of Morality : The Church, the State, and Reproductive Rights in Postsocialist Poland, Athens, OH, Ohio University Press, 2015.

${ }^{50}$ La Fondation polonaise Helsinki pour les droits de l'homme (FHDH). Fondation créée en 1989 pour continuer le travail du Comité Helsinki, passé à la clandestinité en 1982.

${ }^{51}$ S. Wandan, Making New Rights: Constitutional Agenda-Setting in the Transitions of Poland (1989-1997) and South Africa (1990-1994 (1996), Thèse de doctorat, Graduate Faculty of Political and Social Science of the New School, New York, 2004, p. 171-181. 
milieux catholiques conservateurs constituent la principale opposition à toute tentative de soutenir les communautés LGBTQ en Pologne.

\section{Conclusion}

Le changement qui s'est produit dans le discours sexologique sur l'homosexualité dans la Pologne socialiste n'a pas eu lieu par magie. Comme nous l'avons montré, il était intrinsèquement lié à l'évolution de la sexologie polonaise. Parallèlement, ce changement est profondément enraciné dans la dynamique de la guerre froide et dépendait du flux des idées qui parvenaient à passer à travers le rideau de fer. À la différence de ce qui se passait dans d'autres contextes culturels, dans les années 1970, les sexologues polonais étaient formés à envisager la sexualité à partir d'une vaste perspective sociale et culturelle. Les théories sexologiques n'étaient pas élaborées à partir de recherches cliniques, mais à partir des contacts directs des médecins avec leurs patients et leurs lecteurs/correspondants. Par conséquent, les écrits sexologiques de l'époque regorgeaient de témoignages directs de personnes, y compris d'homosexuels, qui s'adressaient par courrier aux sexologues. Les réponses que les sexologues donnaient à ces lettres s'ancraient aussi bien dans un contexte culturel et politique que dans le discours ambiant. Ces sexologues montraient une ouverture intellectuelle aux problèmes de leurs patients et, grâce à leur approche humaniste, ils parvenaient à sympathiser avec eux, avec leurs problèmes et leurs préoccupations. Les homosexuels, patients et lecteurs, demandaient la « réhabilitation » de l'homosexualité. Dès le début des années 1970, les sexologues polonais leur transmettaient des informations sur les progrès des droits des homosexuel·les à l'Ouest (grâce à leurs contacts avec des organisations de lutte pour les droits sexuels de l'Ouest) et laissaient penser que l'homosexualité pouvait être socialement acceptable. Cependant, afin d'échapper à la censure et de correspondre au climat socioculturel, ils ne pouvaient aborder ouvertement par écrit cet aspect. Tout changea dans la seconde moitié des années 1970, quand le discours des droits de la personne commença à se répandre du fait de l'effet Helsinki. L'État ne pouvait plus réprimer violemment l'opposition démocratique puisque les accords d'Helsinki posaient comme condition à l'aide financière aux pays comme la Pologne le respect des droits de la personne et des droits civiques. Les accords d'Helsinki ne mentionnaient cependant pas les droits sexuels. C'est l'ouverture d'esprit des sexologues face à leurs patients, telle qu'elle s'inscrivait dans leur approche de la sexologie, qui les amena à écrire sur les droits homosexuels. Quelques-uns des homosexuels, aujourd'hui âgés, à qui nous avons parlé nous ont dit n'avoir jamais oublié, jusqu'à ce jour, ces écrits, leur importance et le réconfort qu'ils leur apportaient.

En même temps, l'effet Helsinki conduisit à une alliance entre l'Église catholique et l'opposition démocratique. À la différence des droits sexuels, la liberté de religion figurait nommément dans l'accord final d'Helsinki. Sur la question des droits homosexuels, l'effet Helsinki est ambigu. D'une part, l'accord final a contribué grandement au développement de ces droits, mais, d'autre part, il a joué un rôle crucial dans la formation de l'alliance entre l'Église catholique et l'opposition démocratique qui, à long terme, eut pour conséquence la restriction des droits sexuels en Pologne. 


\section{Bibliographie}

Bayer, R., Homosexuality and American Psychiatry: The Politics of Diagnosis, Princeton, NJ, Princeton University Press, 1981.

Dehnert, G., « The Polish Opposition, the Crisis of the Gierek Era, and the Helsinki Process ", in EcKel, J. et Moyn, S. (dir.), The Breakthrough Human Rights in the 1970s, Philadelphia, University of Pennsylvania Press, 2014, p. 166-185.

DodziUK, A., «W obronie zdrowego rozsądku », Tygodnik Mazowsze, 227, 1987, p. 4.

Donert, C., «Whose Utopia? Gender, Ideology, and Human Rights at the 1975 World Congress of Women in East Berlin », in Eckel, J. et Moyn, S. (dir.), The Breakthrough Human Rights in the 1970s, Philadelphia, University of Pennsylvania Press, 2014, p. 68-87.

Donham, D. L., «Freeing South Africa: The "Modernization” of Male-Male Sexuality in Soweto », Cultural Anthropology, 13/1, 1998, p. 3-21.

Essig, L., Queer in Russia, Durham, NC, Duke University Press, 1999.

Fiedotow, A., « Początki ruchu gejowskiego w Polsce », in KulA, M. (dir.), Kłopoty z seksem w PRL. Rodzenie nie całkiem po ludzku, aborcja, choroby, odmienności, Varsovie, Wydawnictwa Uniwersytetu Warszawskiego, Instytut Pamięci Narodowej, 2012, p. 271-272.

Ghodsee, K., « Rethinking State Socialist Mass Women's Organizations. The Committee of the Bulgarian Women's Movement and the United Nations Decade for Women, 1975-1985 », Journal of Women's History, 24/4, 2012, p. 49-73.

Healey, D., Homosexual Desire in Revolutionary Russia, Chicago, University of Chicago Press, 2001.

KościańsKa, A., " Beyond Viagra: Sex Therapy in Poland », Sociologický časopis/ Czech Sociological Review 50/6, 2014, p. 919-938.

KoŚCIAŃSKA, A., "Sex on equal terms? Polish sexology on women's emancipation and "good sex" from the 1970s to present », Sexualities, 19/1-2, 2016, p. 236-256.

KościańsKa, A., Pteć, przyjemność i przemoc. Ksztaltowanie wiedzy eksperckiej o seksualności w Polsce, Varsovie, Wydawnictwa Uniwersytetu Warszawskiego, 2014.

KościańsKa, A., Zobaczyć łosia. Historia polskiej edukacji seksualnej od pierwszej lekcji do internetu, Wołowiec, Czarne, 2017.

KuRIMAY, A. et TAKÁCS, J., « Emergence of the Hungarian homosexual movement in late refrigerator socialism », Sexualities, 20/5-6, 2017, p. 585-603.

Lew-Starowicz, Z., Pan od seksu. Autobiografia najslynniejszego polskiego seksuologa, Cracovie, Znak, 2013.

McLellan, J., Love in the Time of Communism Intimacy and Sexuality in the GDR, Cambridge, Cambridge University Press, 2011.

Mishtal, J., The Politics of Morality: The Church, the State, and Reproductive Rights in Postsocialist Poland, Athens, OH, Ohio University Press, 2015.

Moyn, S., « The Return of the Prodigal: The 1970s as a Turning Point in Human Rights History ", in EcKel, J. et MoYN, S. (dir.), The Breakthrough Human Rights in the 1970s, Philadelphia, University of Pennsylvania Press, 2014. 
OwczarzaK, J., « Defining HIV Risk and Determining Responsibility in Postsocialist Poland », Medical Anthropology Quarterly, 23/4, 2009, p. 417-435.

PŁATEK, M., « Sytuacja osób homoseksualnych w prawie karnym », in WIERUSZEWSKI, R. et WyrzyKowski, M. (dir.), Orientacja seksualna $i$ tożsamość płciowa, Varsovie, Instytut Wydawniczy EuroPrawo, 2009, p. 49-81.

Richardson-LitTle, N., « Dictatorship and Dissent: Human Rights in East Germany in the 1970s », in ECKEL, J. et MoYN, S. (dir.), The Breakthrough Human Rights in the 1970s, Philadelphia, University of Pennsylvania Press, 2014, p. 49-67.

Sokoluk, W., Andziak, D. et Trawińska, M., Przysposobienie do życia w rodzinie, Varsovie, Wydawnictwa Szkolne i Pedagogiczne, 1987.

Stella, F., Lesbian Loves in Soviet and Post-Soviet Russia, Londres, Palgrave Macmillan, 2015.

Szulc, L., Transnational Homosexuals in Communist Poland: Cross-Border Flows in Gay and Lesbian Magazines, New York, Palgrave Macmillan, 2018.

Wandan, S., Making New Rights: Constitutional Agenda-Setting in the Transitions of Poland (1989-1997) and South Africa (1990-1994 (1996), Thèse de doctorat, Graduate Faculty of Political and Social Science of the New School, New York, 2004.

Warkocki, B., « Trzy fale emancypacji homoseksualnej w Polsce », Porównania, 15, 2014, p. 121-132. 